\title{
Pacific
}

Journal of

Mathematics

\section{ON THE CAUCHY PROBLEM FOR A SINGULAR PARABOLIC} EQUATION

\author{
XIANGSHENG XU
}

Volume $174 \quad$ No. 1 


\title{
ON THE CAUCHY PROBLEM FOR A SINGULAR PARABOLIC EQUATION
}

\author{
XIANGSHENG XU
}

The existence of a renormalized solution is established for the Cauchy problem for the parabolic P-Laplacain equation in which $p$ is allowed to be close to 1 and the initial data are only assumed to be locally integrable.

\section{Introduction.}

We shall be concerned with the existence of a solution to the following problem

$$
\begin{gathered}
\frac{\partial}{\partial t} u-\operatorname{div}\left(|\nabla u|^{p-2} \nabla u\right)=0 \quad \text { in } \Sigma_{T} \equiv \mathbf{R}^{N} \times(0, T), \\
u(x, 0)=u_{0}(x) \text { on } \mathbf{R}^{N}
\end{gathered}
$$

in the case where $T>0,1<p<2$, and $u_{0} \in L_{\text {loc }}^{1}\left(\mathbf{R}^{N}\right)$. The restriction on $p$ makes the equation (1.1a) singular because the term $|\nabla u|^{p-2}$, which measures the modulus of ellipticity of the principal part of (1.1a), is unbounded at points where $|\nabla u|$ is 0 . Thus we are dealing with a singular parabolic problem.

It is observed in $[\mathbf{D H}]$ that in the generality considered here an estimate of the form

$$
|\nabla u| \in L_{\mathrm{loc}}^{q}\left(\Sigma_{T}\right), \quad q \geq 1
$$

is no longer possible. This suggests that solutions of (1.1a) display new phenomena that cannot be incorporated into the classical weak formulation. To define our notion of a weak solution, we follow the approach adopted in [X1]. Let $\mathcal{A}=\left\{\theta \in C(\mathbf{R}): \theta\right.$ is a Lipschitz function whose derivative $\theta^{\prime}(s)$ exists except at finitely many points and $\theta^{\prime}(s)=0$ for $|s|$ sufficiently large $\}$. If a measurable function $v$ on $\Sigma_{T}$ is such that $\theta(v) \in L^{p}\left(0, T ; W_{\text {loc }}^{1, p}\left(\mathbf{R}^{N}\right)\right)$ for all $\theta \in \mathcal{A}$, then we can define a measurable function $g: \Sigma_{T} \rightarrow \mathbf{R}^{N}$ so that

$$
g=\nabla P_{M}(v) \text { almost everywhere on }\{|v|<M\}
$$

for all $M>0$, where $P_{M}(s)=\min \{|s|, M\} \operatorname{sign}(s)$. The function $g$ is viewed as the spatial gradient of $v$, and is also denoted by $\nabla v$. We are ready to present our definition of a solution.

Definition. A measurable function $u$ on $\Sigma_{T}$ is said to be a renormalized solution of (1.1) if:

1. $u \in C\left([0, T] ; L_{\text {loc }}^{1}\left(\mathbf{R}^{N}\right)\right)$; 
2. For each $\theta \in \mathcal{A}, \theta(u) \in L^{p}\left(0, T ; W_{\mathrm{loc}}^{1, p}\left(\mathbf{R}^{N}\right)\right)$ and $\nabla \theta(u)=\theta^{\prime}(u) \nabla u$ almost everywhere on $\Sigma_{T}$, where $\theta^{\prime}(u)$ is understood to be 0 if $u \in$ $B_{\theta} \equiv\left\{s \in \mathbf{R}: \theta^{\prime}(s)\right.$ does not exist $\}$

3. $|\nabla u|^{p-1} \in L^{1}\left(0, T ; L_{\text {loc }}^{1}\left(\mathbf{R}^{N}\right)\right)$ and

$$
\begin{aligned}
& -\int_{\Sigma_{T}} \int_{0}^{u} \theta(s) d s \varphi_{t} d x d t+\int_{\Sigma_{T}}|\nabla u|^{p-2} \nabla u(\nabla \theta(u) \varphi+\theta(u) \nabla \varphi) d x d t \\
& =\int_{\mathbf{R}^{N}} \varphi(x, 0) \int_{0}^{u_{0}(x)} \theta(s) d s d x
\end{aligned}
$$

for all $\theta \in \mathcal{A}$ and all $\varphi \in C_{0}^{\infty}\left(\mathbf{R}^{N} \times(-\infty, T)\right)$.

The idea of a renormalized solution was originated in the study of the Boltzmann equation; see [DL1, DL2] for details. An elliptic version of this idea appears in [BGDM]. The definition here is a slight modification of that in [X1]; also see [X2] where it is evident that the notion of a renormalized solution is the correct notion of solution for p-Laplacian problems. The objective of this paper is to show that there exists a renormalized solution to $(1.1)$.

If $u_{0} \geq 0$, the existence and uniqueness of a solution to (1.1) are established in [DH]. In [X1], the sign restriction on $u_{0}$ is removed, but $R^{N}$ is replaced with a bounded domain $\Omega$. The stationary problem is considered in [X2] and references therein. The question of existence and uniqueness of a solution to (1.1) in the case where $u_{0}$ may change sign was proposed as an open problem in $[\mathbf{D H}]$. In this paper, we solve the question of existence, while the question of uniqueness remains open.

It is interesting to note that we obtain a renormalized solution to (1.1) without imposing any growth condition on $u_{0}$. This is in sharp contrast with the case $p>2$ [D]. Also, it is easy to infer from the argument in $\left[\mathbf{D}\right.$, p. 188-192] that if $u_{0} \in L^{s}\left(\mathbf{R}^{N}\right), s=N(2-p) / p, 1<p<2 N /(N+1)$, and $N \geq 2$, then the renormalized solution $u$ constructed here will extinct in finite time, i.e., there exists a positive number $T^{*}$ such that $u(x, t)=0$ for all $t>T^{*}$.

The main gap between the case $u_{0} \geq 0$, and the case where $u_{0}$ may change sign, is that in the latter case an estimate of the type

$$
\int_{s}^{T} \int_{\{|x|<R\}} \frac{u_{t}^{2}}{(1+|u|)^{1+\varepsilon}} d x d t<\infty, \quad s \in(0, T), \varepsilon>0, R>0
$$

is no longer available. To overcome this difficulty, we develop an analysis that combines the best features of the arguments in [DH] and [X1] with a compactness theorem of Simon [S]. 
This work is organized as follows. In Section 2, we prove a comparison principle for classical weak solutions of (1.1a). This result is used in Section 3 to prove the existence of a renormalized solution.

We conclude this section by making some remarks on notation. Let $R>0$, and we denote by $B_{R}$ the ball centered at the origin with radius $R$. Fix $R>r>0$. We say that $\xi$ is a cut-off function associated with $R$ and $r$ if $\xi \in C_{0}^{\infty}\left(B_{R}\right), 0 \leq \xi \leq 1, \xi=1$ on $B_{r}$, and $|\nabla \xi| \leq \frac{2}{R-r}$. Let $E$ be a measurable set in $\mathbf{R}^{N+1}$. We use $|E|$ to denote the Lebesque measure of $E$.

\section{Preliminaries.}

In this section we consider the problem

$$
\begin{aligned}
\frac{\partial}{\partial t} u-\operatorname{div}\left(|\nabla u|^{p-2} \nabla u\right) & =0 \quad \text { in } \Sigma_{T}, \\
u(x, 0) & =u_{0}(x) \text { on } \mathbf{R}^{N}
\end{aligned}
$$

in the case where $u_{0} \in L_{\text {loc }}^{2}\left(\mathbf{R}^{N}\right)$ and $1<p<2$. A function $u$ on $\Sigma_{T}$ is said to be a classical weak solution of $(2.1)$ if:

(i) $u \in C\left([0, T] ; L_{\text {loc }}^{2}\left(\mathbf{R}^{N}\right)\right) \cap L^{p}\left(0, T ; W_{\text {loc }}^{1, p}\left(\mathbf{R}^{N}\right)\right)$;

(ii) $-\int_{\Sigma_{T}} u \varphi_{t} d x d t+\int_{\Sigma_{T}}|\nabla u|^{p-2} \nabla u \nabla \varphi d x d t=\int_{\mathbf{R}^{N}} \varphi(x, 0) u_{0}(x) d x$ for all $\varphi \in C_{0}^{\infty}\left(\mathbf{R}^{N} \times(-\infty, T)\right)$.

Let $u$ be a classical weak solution to (2.1). Then we can easily deduce from (ii) that for each $\rho>0$,

$$
u_{t} \in L^{p^{\prime}}\left(0, T ; W^{-1, p^{\prime}}\left(B_{\rho}\right)\right)
$$

$$
u_{t}-\operatorname{div}\left(|\nabla u|^{p-2} \nabla u\right)=0 \text { in } W^{-1, p^{\prime}}\left(B_{\rho}\right) \text { for almost every } t \in(0, T) .
$$

Here and in what follows $p^{\prime}=p /(p-1)$.

Lemma 2.1. Let $u$ be a classical weak solution of (2.1). Then $u_{0} \in$ $L_{\text {loc }}^{\infty}\left(\mathbf{R}^{N}\right)$ implies $u \in L^{\infty}\left(0, T ; L_{\text {loc }}^{\infty}\left(\mathbf{R}^{N}\right)\right)$.

Remark. If $u_{0} \geq 0$, then this lemma is a direct consequence of Theorem III.6.2 in [DH].

Proof of Lemma 2.1. We modify a device in $[\mathbf{D H}]$. Fix $R>0$. For $n=$ $0,1,2, \ldots$, define

$$
\rho_{n}=R\left(1+2^{-n}\right), B_{n}=B_{\rho_{n}}, k_{n}=M\left(2-2^{-n}\right),
$$


where $M \geq\left\|u_{0}\right\|_{L^{\infty}\left(B_{2 R}\right)}$ will be selected later. Let $\xi_{n}$ be a cut-off function associated with $\rho_{n}$ and $\rho_{n+1}$. Then we can derive from the chain rule [X1] that the function $t \rightarrow \frac{1}{2} \int_{B_{n}}\left[\left(u-k_{n}\right)^{+}\right]^{2} \xi_{n}^{p} d x$ is absolutely continuous on $[0 . T]$, and

$$
\frac{d}{d t} \frac{1}{2} \int_{B_{n}}\left[\left(u-k_{n}\right)^{+}\right]^{2} \xi_{n}^{p} d x=\left(u_{t},\left(u-k_{n}\right)^{+} \xi_{n}^{p}\right)
$$

almost everywhere on $(0, T)$,

where $(\cdot, \cdot)$ denotes the duality pairing between $W^{-1, p^{\prime}}\left(B_{n}\right)$ and $W_{0}^{1, p}\left(B_{n}\right)$. Keep this in mind, use $\left(u-k_{n}\right)^{+} \xi_{n}^{p}$ as a test function in (2.3), thereby obtain

$$
\begin{aligned}
& \frac{d}{d t} \frac{1}{2} \int_{B_{n}}\left[\left(u-k_{n}\right)^{+}\right]^{2} \xi_{n}^{p} d x+\int_{B_{n}}\left|\nabla\left(u-k_{n}\right)^{+}\right|^{p} \xi_{n}^{p} d x \\
& =-\int_{B_{n}}\left|\nabla\left(u-k_{n}\right)^{+}\right|^{p-2} \nabla\left(u-k_{n}\right)^{+}\left(u-k_{n}\right)^{+} p \xi_{n}^{p-1} \nabla \xi_{n} d x \\
& \leq \frac{1}{2} \int_{B_{n}}\left|\nabla\left(u-k_{n}\right)^{+}\right| \xi_{n}^{p} d x+2^{p-1}\left(\frac{p}{R}\right)^{p} 2^{p(n+1)} \int_{B_{n}}\left[\left(u-k_{n}\right)^{+}\right]^{p} d x .
\end{aligned}
$$

Consequently,

$$
\begin{aligned}
& \max _{0 \leq t \leq T} \int_{B_{n}}\left[\left(u-k_{n}\right)^{+}\right]^{2} \xi_{n}^{p} d x+\int_{B_{n} \times(0, T)}\left|\nabla\left(u-k_{n}\right)^{+}\right|^{p} \xi_{n}^{p} d x d t \\
& \leq\left(\frac{p}{R}\right)^{p} 2^{p(n+2)} \int_{B_{n} \times(0, T)}\left[\left(u-k_{n}\right)^{+}\right]^{p} d x d t .
\end{aligned}
$$

This, in conjunction with the Gagliardo-Nirenberg-Sobolev inequality, implies

$$
\begin{aligned}
& \int_{B_{n} \times(0, T)}\left[\left(u-k_{n}\right)^{+} \xi_{n}\right]^{p \frac{N+2}{N}} d x d t \\
& \leq c_{0}\left(\sup _{0 \leq t \leq T} \int_{B_{n}}\left[\left(u-k_{n}\right)^{+} \xi_{n}\right]^{2} d x\right)^{\frac{p}{N}} \\
& \cdot \int_{B_{n} \times(0, T)}\left|\nabla\left(\left(u-k_{n}\right)^{+} \xi_{n}\right)\right|^{p} d x d t \\
& \leq c_{1} \frac{2^{\left(\frac{p(N+p)}{N}\right) n}}{R^{\frac{p(N+p)}{N}}}\left(\int_{B_{n} \times(0, T)}\left[\left(u-k_{n}\right)^{+}\right]^{p} d x d t\right)^{\frac{(N+p)}{N}} .
\end{aligned}
$$

Here, and in what follows, $c_{i}, i \in\{0,1,2, \ldots\}$, denote positive constants depending only upon $p, N$. We estimate

$$
\int_{B_{n+1} \times(0, T)}\left[\left(u-k_{n+1}\right)^{+}\right]^{p} d x d t
$$




$$
\begin{aligned}
\leq & \int_{B_{n} \times(0, T)}\left[\left(u-k_{n+1}\right)^{+} \xi_{n}\right]^{p} d x d t \\
\leq & \left|B_{n} \times(0, T) \cap\left\{u>k_{n+1}\right\}\right|^{\frac{2}{N+2}} \\
& \cdot\left(\int_{B_{n} \times(0, T)}\left[\left(u-k_{n+1}\right)^{+} \xi_{n}\right]^{p \frac{N+2}{N}} d x d t\right)^{\frac{N}{N+2}} \\
\leq & c_{2} \frac{2^{\left(\frac{p(N+p)}{(N+2)}\right) n}}{R^{\frac{p(N+p)}{(N+2)}}\left|B_{n} \times(0, T) \cap\left\{u>k_{n+1}\right\}\right|^{\frac{2}{N+2}}} \\
& \cdot\left(\int_{B_{n} \times(0, T)}\left[\left(u-k_{n}\right)^{+}\right]^{p} d x d t\right)^{\frac{N+p}{N+2}} .
\end{aligned}
$$

Observe that

$$
\begin{aligned}
& \int_{B_{n} \times(0, T)}\left[\left(u-k_{n}\right)^{+}\right]^{p} d x d t \\
& \geq \int_{B_{n} \times(0, T) \cap\left\{u>k_{n+1}\right\}}\left(k_{n+1}-k_{n}\right)^{p} d x d t \\
& =M^{p} 2^{-p(n+1)}\left|B_{n} \times(0, T) \cap\left\{u>k_{n+1}\right\}\right| .
\end{aligned}
$$

This, together with (2.6) shows that

$$
\begin{aligned}
\int_{B_{n+1} \times(0, T)} & {\left[\left(u-k_{n+1}\right)^{+}\right]^{p} d x d t } \\
& \leq c_{3} \frac{2^{\left[\frac{p(N+p)}{(N+2)}+\frac{2 p}{(N+2)}\right] n}}{R^{\frac{p(N+p)}{(N+2)}} M^{\frac{2 p}{(N+2)}}}\left(\int_{B_{n} \times(0, T)}\left[\left(u-k_{n}\right)^{+}\right]^{p} d x d t\right)^{1+\frac{p}{N+2}} .
\end{aligned}
$$

According to a result in [LSU, p. 95], $\lim _{n \rightarrow \infty} \int_{B_{n} \times(0, t)}\left[\left(u-k_{n}\right)^{+}\right]^{p} d x d t=$ 0, provided we can select $M \geq\left\|u_{0}\right\|_{L^{\infty}\left(B_{2 R}\right)}$ so that

$$
\begin{aligned}
& \int_{B_{2 R} \times(0, T)}\left[(u-M)^{+}\right]^{p} d x d t \leq\left(\frac{c_{3}}{R^{\frac{p(N+p)}{(N+2)}} M^{\frac{2 p}{(N+2)}}}\right)^{-\frac{N+2}{p}} \\
& \cdot\left(2^{\frac{\left(p N+p^{2}+2 p\right)}{(N+2)}}\right)^{-\left(\frac{N+2}{p}\right)^{2}} \\
& \leq c_{4} R^{(N+p)} M^{2} \text {. }
\end{aligned}
$$

This can be easily done, and hence

$$
\int_{B_{R} \times(0, T)}\left[(u-2 M)^{+}\right]^{p} d x d t \leq \lim _{n \rightarrow \infty} \int_{B_{R} \times(0, T)}\left[\left(u-k_{n}\right)^{+}\right]^{p} d x d t=0 .
$$


To see that $u$ is also bounded below, note that $v=-u$ is a classical weak solution of the following problem

$$
\begin{aligned}
\frac{\partial v}{\partial t}-\operatorname{div}\left(|\nabla v|^{p-2} \nabla v\right) & =0 \text { in } \Sigma_{T} \\
v(x, 0) & =-u_{0}(x) \text { in } \mathbf{R}^{N}
\end{aligned}
$$

This completes the proof of the lemma.

Before we continue, let us recall the following lemma from [O, pp. 145-147].

Lemma 2.2. Let $x, y$ be any two vectors in $\mathbf{R}^{N}$ and $p \in(1,2]$. Then,

(a) $\left(|x|^{p-2} x-|y|^{p-2} y\right)(x-y) \geq(p-1) \frac{|x-y|^{2}}{(|x|+|y|)^{2-p}}$;

(b) $\left.|| x\right|^{p-2} x-|y|^{p-2} y|\leq \sqrt{5}| x-\left.y\right|^{p-1}$.

Lemma 2.3. Let $u_{0}, v_{0}$ be two functions in $L_{\text {loc }}^{\infty}\left(\mathbf{R}^{N}\right)$. Assume that $u$ and $v$ are classical weak solutions of (2.1a) with initial conditions $u_{0}$ and $v_{0}$, respectively. Then $u_{0} \leq v_{0}$ implies $u \leq v$.

Proof. Fix $R>r>0$. Let $\xi$ be a cut-off function associated with $R$ and $r$. By Lemma $2.2, u, v \in L^{\infty}\left(0, T ; L_{\text {loc }}^{\infty}\left(\mathbf{R}^{N}\right)\right)$. Thus for each $q>1,\left[(u-v)^{+}\right]^{q} \xi^{2} \in$ $L^{p}\left(0, T ; W_{0}^{1, p}\left(B_{R}\right)\right)$. We can conclude from $(2.3)$ and the chain rule $[\mathbf{X 1}]$ that

$$
\begin{aligned}
& \frac{d}{d t} \frac{1}{q+1} \int_{B_{R}}\left[(u-v)^{+}\right]^{q+1} \xi^{2} d x \\
& \quad+\int_{B_{R}}\left(|\nabla u|^{p-2} \nabla u-|\nabla u|^{p-2} \nabla v\right) q\left[(u-v)^{+}\right]^{q-1} \nabla(u-v) \xi^{2} d x \\
& =-\int_{B_{R}}\left(|\nabla u|^{p-2} \nabla u-|\nabla v|^{p-2} \nabla v\right)\left[(u-v)^{+}\right]^{q} 2 \xi \nabla \xi d x \\
& \leq\left.\frac{2}{R-r} \int_{B_{R}}|| \nabla u\right|^{p-2} \nabla u-|\nabla v|^{p-2} \nabla v \mid\left[(u-v)^{+}\right]^{q} \xi d x .
\end{aligned}
$$

Set

$$
A_{t}=\left\{x:(u(x, t)-v(x, t))^{+} \frac{2}{R-r} \leq \frac{1}{2} q\left|\nabla(u(x, t)-v(x, t))^{+}\right| \xi(x)\right\} .
$$

We compute, with the aid of Lemma 2.2, that

$$
\left.\frac{2}{R-r} \int_{B_{R}}|| \nabla u\right|^{p-2} \nabla u-|\nabla v|^{p-2} \nabla v \mid\left[(u-v)^{+}\right]^{q} \xi d x
$$




$$
\begin{aligned}
\leq & \left.\frac{1}{2} \int_{B_{R} \cap A_{t}}|| \nabla u\right|^{p-2} \nabla u-|\nabla v|^{p-2} \nabla v|| \nabla(u-v)^{+} \mid q\left[(u-v)^{+}\right]^{q-1} \xi^{2} d x \\
& +\frac{2}{R-r} \int_{B_{R} \backslash A_{t}} \sqrt{5}|\nabla u-\nabla v|^{p-1}\left[(u-v)^{+}\right]^{q} \xi d x \\
\leq & \frac{1}{2} \int_{B_{R}}\left(|\nabla u|^{p-2} \nabla u-|\nabla v|^{p-2} \nabla v\right) \nabla(u-v) q\left[(u-v)^{+}\right]^{q-1} \xi^{2} d x \\
& +\frac{2}{R-r} \int_{B_{R}} \sqrt{5}\left(\frac{4}{q(R-r)}(u-v)^{+}\right)^{p-1}\left[(u-v)^{+}\right]^{q} d x
\end{aligned}
$$

Use this in (2.8) to obtain

$$
\int_{B_{r}}\left[(u-v)^{+}\right]^{q+1} d x \leq \frac{\sqrt{5}(q+1) 2^{2 p-1}}{q^{p-1}(R-r)^{p}} \int_{B_{R} \times(0, t)}\left[(u-v)^{+}\right]^{q+p-1} d x d \tau .
$$

Now we are ready to employ an argument in $[\mathbf{D H}]$. Fix $\rho>0$, and set

$$
\begin{aligned}
\rho_{n} & =\left(\sum_{i=0}^{n} 2^{-i}\right) \rho, \quad B_{n}=B_{\rho_{n}}, \\
\Lambda_{n} & =\sup _{0 \leq T \leq t} \int_{B_{n}}\left[(u-v)^{+}\right]^{q+1} d x \quad(n=0,1,2, \ldots) .
\end{aligned}
$$

We can infer from (2.9) that

$$
\begin{aligned}
\Lambda_{n} & \leq c \frac{2^{p(n+1)}}{\rho^{p}} \int_{B_{n+1} \times(0, t)}\left[(u-v)^{+}\right]^{q+p-1} d x d \tau \\
& \leq c t^{\frac{2-p}{q+1}+1}(2 p)^{N \frac{2-p}{q+1}} \Lambda_{n+1}^{\frac{q+p-1}{q+1}} \frac{2^{p(n+1)}}{\rho^{p}} \\
& =c_{1} t^{\frac{3-p+q}{q+1}} \frac{2^{p n}}{\rho^{p-\frac{(2-p) N}{(q+1)}}} \Lambda_{n+1}^{\frac{(q+p-1)}{(+1)}} \\
& \leq \delta \Lambda_{n+1}+\left(2^{p \frac{q+1}{2-p}}\right)^{n} c(\delta)\left(\frac{t^{\frac{(3-p+q)}{(q+1)}}}{\rho^{p-\frac{(2-p) N}{(q+1)}}}\right)^{\frac{q+1}{2-p}} .
\end{aligned}
$$

Here $\delta>0$ is arbitrary. This implies

$$
\Lambda_{0} \leq \delta^{n} \Lambda_{n}+\frac{1}{\delta} c(\delta)\left(\frac{t^{\frac{(3-p+q)}{(q+1)}}}{\rho^{p-\frac{(2-p) N}{(q+1)}}}\right)^{\frac{q+1}{2-p}} \sum_{i=0}^{n+1}\left(\delta 2^{p \frac{q+1}{2-p}}\right)^{i}
$$

Now we select $\delta>0$ and $q>0$ so that

$$
\delta 2^{p \frac{q+1}{2-p}}=\frac{1}{2} \quad \text { and } \quad(q+1) p-(2-p) N>0 .
$$


We conclude from (2.10) that

$$
\begin{gathered}
\sup _{0 \leq \tau \leq t} \int_{B_{\rho}}\left[(u-v)^{+}\right]^{q+1} d x \leq c\left(\frac{t^{\frac{(3-p+q)}{(q+1)}}}{\rho^{\frac{(q+1) p-(2-p) N}{q+1}}}\right)^{\frac{q+1}{2-p}} \\
\rightarrow 0 \quad \text { as } \quad \rho \rightarrow \infty .
\end{gathered}
$$

This proves the lemma.

An easy consequence of Lemma 2.1 and Lemma 2.3 is that

$$
\|u(\cdot, t)\|_{L^{\infty}\left(\mathbf{R}^{N}\right)} \leq\left\|u_{0}\right\|_{L^{\infty}\left(\mathbf{R}^{N}\right)}
$$

for each $t>0$.

\section{Existence.}

The main result of this section is:

Theorem 3.1. Assume that $u_{0} \in L_{\text {loc }}^{1}\left(\mathbf{R}^{N}\right)$, and $1<p<2$. Then there exists a renormalized solution to (1.1).

Proof. If $k \in\{1,2, \ldots\}$, define

$$
\begin{aligned}
& f_{k}(x)=\min \left\{u_{0}^{+}(x), k\right\}, \\
& g_{k}(x)=\min \left\{u_{0}^{-}(x), k\right\} .
\end{aligned}
$$

For each $k$, consider the approximating problem

$$
\begin{array}{rr}
\frac{\partial u_{k}}{\partial t}-\operatorname{div}\left(\left|\nabla u_{k}\right|^{p-2} \nabla u_{k}\right)=0 & \text { on } \quad \Sigma_{T} \\
u(x, 0)=u_{0 k}(x)=f_{k}-g_{k} & \text { in } \mathbf{R}^{N} .
\end{array}
$$

The existence of a classical weak solution to (3.3) can be inferred from a result in [DH, D]. Since $u_{0 k} \in L^{\infty}\left(\mathbf{R}^{N}\right)$, Lemma 2.3 asserts the uniqueness. The remaining proof is divided into several lemmas.

Lemma 3.1. For each $\rho>0$, there exists a $c(\rho)>0$ such that

$$
\begin{aligned}
& \max _{0 \leq t \leq T} \int_{B_{\rho}}\left|u_{k}(x, t)\right| d x \leq c(\rho), \\
& \int_{B_{\rho} \times(0, T)}\left|\nabla u_{k}\right|^{p-1} d x d t \leq c(\rho)(k=1,2, \ldots) .
\end{aligned}
$$


Proof. For each $k$, let $v_{k}$ be the classical weak solution of the following problem

$$
\begin{array}{r}
\frac{\partial}{\partial t} v_{k}-\operatorname{div}\left(\left|\nabla v_{k}\right|^{p-2} \nabla v_{k}\right)=0 \quad \text { in } \quad \Sigma_{T} \\
v_{k}(x, 0)=f_{k}(x) \quad \text { on } \quad \mathbf{R}^{N}
\end{array}
$$

and $w_{k}$ be the classical weak solution of the following problem

$$
\begin{array}{r}
\frac{\partial}{\partial t} w_{k}-\operatorname{div}\left(\left|\nabla w_{k}\right|^{p-2} \nabla w_{k}\right)=0 \quad \text { in } \quad \Sigma_{T} \\
w_{k}(x, 0)=-g_{k}(x) \quad \text { on } \quad \mathbf{R}^{N} .
\end{array}
$$

In light of Lemma 2.3, we have

$$
w_{k} \leq u_{k} \leq v_{k} \quad \text { almost everywhere on } \Sigma_{T}
$$

for all $k$. Since $f_{k} \geq 0$ on $\mathbf{R}^{N}$, we can invoke a result in [DH, p. 260] to obtain that there exists a $c_{1}(\rho)>0$ such that

$$
\max _{0 \leq t \leq T} \int_{B_{\rho}} v_{k}(x, t) d x \leq c_{1}(\rho) \quad(k=1,2, \ldots) .
$$

Note that $z_{k}=-w_{k}$ is the classical weak solution of the problem

$$
\begin{array}{r}
\frac{\partial}{\partial t} z_{k}-\operatorname{div}\left(\left|\nabla z_{k}\right|^{p-2} \nabla z_{k}\right)=0 \quad \text { in } \quad \Sigma_{T} \\
z_{k}(x, 0)=g_{k}(x) \quad \text { on } \quad \mathbf{R}^{N} .
\end{array}
$$

Thus, we can find $c_{2}(\rho)>0$ with

$$
\max _{0 \leq t \leq T} \int_{B_{\rho}}\left|w_{k}(x, t)\right| d x \leq c_{2}(\rho) \quad(k=1,2, \ldots) .
$$

We see that (3.4) is a consequence of (3.8), (3.9), and (3.10). To see (3.5), for each $\varepsilon>0$ define

$$
\phi_{\varepsilon}(s)= \begin{cases}1-\frac{1}{(1+s)^{\varepsilon}} & \text { if } s \geq 0 \\ -\phi_{\varepsilon}(-s) & \text { if } s<0\end{cases}
$$

Let $\xi$ be a cut-off function associated with $2 \rho$ and $\rho$. Then using $\phi_{\varepsilon}\left(u_{k}\right) \xi^{p}$ as a test function in (3.3a), we derive from a standard argument [X1] that

$$
\frac{d}{d t} \int_{B_{2 \rho}} \int_{0}^{u_{k}(x, t)} \phi_{\varepsilon}(s) d s \xi^{p}(x) d x+\int_{B_{2 \rho}} \phi_{\varepsilon}^{\prime}\left(u_{k}\right)\left|\nabla u_{k}\right|^{p} \xi^{p} d x
$$




$$
=-\int_{B_{2 \rho}}\left|\nabla u_{k}\right|^{p-2} \nabla u_{k} \phi_{\varepsilon}\left(u_{k}\right) p \xi^{p-1} \nabla \xi d x .
$$

Note that

$$
\phi_{\varepsilon}^{\prime}=\frac{\varepsilon}{(1+|s|)^{1+\varepsilon}} \quad \text { and } \quad\left|\phi_{\varepsilon}\right| \leq 1
$$

and that

$$
a b \leq \sigma a^{p}+\sigma^{-\frac{p^{\prime}}{p}} b^{p^{\prime}}, a>0, b>0, \sigma>0 .
$$

We deduce from (3.12) that

$$
\begin{aligned}
& \int_{B_{2 \rho}} \int_{0}^{u_{k}(x, t)} \phi_{\varepsilon}(s) d s \xi^{p}(x) d x+\frac{\varepsilon}{2} \int_{B_{2 \rho} \times(0, t)} \frac{\left|\nabla u_{k}\right|^{p} \xi^{p}}{\left(1+\left|u_{k}\right|\right)^{1+\varepsilon}} d x d \tau \\
& \leq \int_{B_{2 \rho}} \int_{0}^{u_{0 k}(x)} \phi_{\varepsilon}(s) d s \xi^{p}(x) d x \\
& \quad+\left(\frac{\varepsilon}{2}\right)^{1-p}\left(\frac{p}{\rho}\right)^{p} \int_{B_{2 \rho} \times(0, t)}\left(1+\left|u_{k}\right|\right)^{(1+\varepsilon)(p-1)} d x d \tau .
\end{aligned}
$$

Observe that $\int_{0}^{u_{k}(x, t)} \phi_{\varepsilon}(s) d s \geq 0$ on $\Sigma_{T}$. Then select $\varepsilon_{0}>0$ so that

$$
\left(1+\varepsilon_{0}\right)(p-1)=1 \text {. }
$$

It follows from (3.14) and (3.4) that there exists a $c(\rho)>0$ with

$$
\int_{B_{\rho} \times(0, T)} \frac{\left|\nabla u_{k}\right|^{p}}{\left(1+\left|u_{k}\right|\right)^{1+\varepsilon_{0}}} d x d t \leq c(\rho) .
$$

We estimate that

$$
\begin{aligned}
\int_{B_{\rho} \times(0, T)}\left|\nabla u_{k}\right|^{p-1} d x d t= & \int_{B_{\rho} \times(0, T)} \frac{\left|\nabla u_{k}\right|^{p-1}}{\left(1+\left|u_{k}\right|\right)^{\frac{\left(1+\varepsilon_{0}\right)}{p^{\prime}}}}\left(1+\left|u_{k}\right|\right)^{\frac{\left(1+\varepsilon_{0}\right)}{p^{\prime}}} d x d t \\
\leq & \frac{\varepsilon_{0}}{2} \int_{B_{\rho} \times(0, T)} \frac{\left|\nabla u_{k}\right|^{p}}{\left(1+\left|u_{k}\right|\right)^{1+\varepsilon_{0}}} d x d t \\
& +\left(\frac{\varepsilon_{0}}{2}\right)^{1-p} \int_{B_{\rho} \times(0, T)}\left(1+\left|u_{k}\right|\right)^{\left(1+\varepsilon_{0}\right)(p-1)} d x d t .
\end{aligned}
$$

This implies (3.5).

Lemma 3.2. For $k \in\{1,2, \ldots\}$, there hold

$$
\int_{B_{\rho} \times(0, T)} \frac{1}{\left(1+\left|u_{k}\right|\right)^{1+\varepsilon}}\left|\nabla u_{k}\right|^{p} d x d t \leq \frac{c(\rho)}{\varepsilon} \quad(\varepsilon>0),
$$




$$
\int_{B_{\rho} \times(0, T) \cap\left\{\left|u_{k}\right| \leq M\right\}}\left|\nabla u_{k}\right|^{p} d x d t \leq M c(\rho) \quad(M>0)
$$

for some $c(\rho)>0$.

Proof. Let $\rho>0$ and $\xi$ be a cut-off function associated with $2 \rho$ and $\rho$. Use $\phi_{\varepsilon}\left(u_{k}\right) \xi$ as a test function in (3.3a) to obtain

$$
\begin{aligned}
& \int_{B_{\rho} \times(0, T)} \frac{\varepsilon}{\left(1+\left|u_{k}\right|\right)^{1+\varepsilon}}\left|\nabla u_{k}\right|^{p} d x d t \\
& \leq \int_{B_{2 \rho}}\left|u_{0}(x)\right| d x+\frac{1}{\rho} \int_{B_{2 \rho} \times(0, T)}\left|\nabla u_{k}\right|^{p-1} d x d t .
\end{aligned}
$$

This, together with (3.5) implies (3.15). To see (3.16), for $M>0$ let $P_{M}(s)$ be given as before. Then use $P_{M}\left(u_{k}\right) \xi$ as a test function in (3.3a) to get

$\int_{B_{\rho} \times(x, T)} P_{M}^{\prime}\left(u_{k}\right)\left|\nabla u_{k}\right|^{p} d x d t \leq M \int_{B_{2 \rho}}\left|u_{0}\right| d x+\frac{M}{\rho} \int_{B_{2 \rho} \times(0, T)}\left|\nabla u_{k}\right|^{p-1} d x d t$.

This completes the proof.

Lemma 3.3. There exists a subsequence of $\left\{u_{k}\right\}$, still denoted by $\left\{u_{k}\right\}$, and a function $u \in L_{\text {loc }}^{1}\left(\mathbf{R}^{N} \times(0, T)\right)$ with

$$
u_{k} \rightarrow u \text { almost everywhere on } \Sigma_{T} \text {. }
$$

Proof. Fix $\rho>0$, and let $\xi$ be given as in the proof of Lemma 3.2. We conclude from (3.3a) that

$$
\begin{aligned}
\int_{0}^{T}\left(\frac{\partial}{\partial t} u_{k}, \frac{1}{1+u_{k}^{2}} \xi \varphi\right) d t & +\int_{B_{2 \rho} \times(0, T)}\left|\nabla u_{k}\right|^{p-2} \nabla u_{k} \nabla \xi \varphi d x d t \\
& +\int_{B_{2 \rho} \times(0, T)} \frac{1}{1+u_{k}^{2}}\left|\nabla u_{k}\right|^{p-2} \nabla u_{k} \xi \nabla \varphi d x d t \\
& -\int_{B_{2 \rho} \times(0, T)} \frac{2 u_{k}}{\left(1+u_{k}^{2}\right)^{2}}\left|\nabla u_{k}\right|^{p} \xi \varphi d x d t=0
\end{aligned}
$$

for all $\varphi \in C_{0}^{\infty}\left(B_{2 \rho} \times(0, T)\right)$. Here, $(\cdot, \cdot)$ denotes the duality pairing between $W^{-1, p^{\prime}}\left(B_{2 \rho}\right)$ and $W_{0}^{1, p}\left(B_{2 \rho}\right)$. We infer from an argument in [X1] that

$\left(\frac{\partial}{\partial t} u_{k}, \frac{1}{1+u_{k}^{2}} \xi \varphi\right)=\left(\frac{\partial}{\partial t}\left(\xi \arctan u_{k}\right), \varphi\right)$ almost everywhere on $(0, T)$. 
This, combined with (3.18) indicates that

$$
\begin{gathered}
\frac{\partial}{\partial t}\left(\xi \arctan u_{k}\right)-\operatorname{div}\left(\frac{1}{1+u_{k}^{2}} \xi\left|\nabla u_{k}\right|^{p-2} \nabla u_{k}\right) \\
+\left|\nabla u_{k}\right|^{p-2} \nabla u_{k} \nabla \xi-\frac{2 u_{k}}{\left(1+u_{k}^{2}\right)^{2}} \xi\left|\nabla u_{k}\right|^{p}=0 \\
\quad \text { in } \quad \mathcal{D}^{\prime}\left(B_{2 \rho} \times(0, T)\right) .
\end{gathered}
$$

Now set

$$
\begin{aligned}
F_{k} & =\operatorname{div}\left(\frac{1}{1+u_{k}^{2}} \xi\left|\nabla u_{k}\right|^{p-2} \nabla u_{k}\right), \\
G_{k} & =-\left|\nabla u_{k}\right|^{p-2} \nabla u_{k} \nabla \xi-\frac{2 u_{k}}{\left(1+u_{k}^{2}\right)^{2}} \xi\left|\nabla u_{k}\right|^{p} .
\end{aligned}
$$

It is easy to verify from (3.5) and (3.15) that

$$
\begin{aligned}
\left\{G_{k}\right\} & \text { is bounded in } L^{1}\left(B_{2 \rho} \times(0, T)\right), \\
\left\{F_{k}\right\} & \text { is bounded in } L^{p^{\prime}}\left(0, T ; W^{-1, p^{\prime}}\left(B_{2 \rho}\right)\right), \\
\left\{\xi \arctan u_{k}\right\} & \text { is bounded in } L^{p}\left(0, T ; W_{0}^{1, p}\left(B_{2 \rho}\right)\right) .
\end{aligned}
$$

This puts us in a position to invoke Lemma 4.2 in [BM] to conclude that

$$
\left\{\xi \arctan u_{k}\right\} \text { is precompact in } L_{\text {loc }}^{p}\left(B_{2 \rho} \times(0, T)\right) \text {. }
$$

In particular, we can extract a subsequence of $\left\{u_{k}\right\}$, still denoted by $\left\{u_{k}\right\}$, such that

$$
\arctan u_{k} \text { converges almost everywhere on } B_{\rho} \times(0, T) .
$$

Note that $u_{k}=\tan \left(\arctan u_{k}\right)$. We may define

$$
u(x, t)=\lim _{k \rightarrow \infty} u_{k}(x, t) \quad \text { for almost everywhere }(x, t) \in B_{\rho} \times(0, T) .
$$

To conclude that $\left\{u_{k}\right\}$ converges almost everywhere on $B_{\rho} \times(0, T)$, we must show that $|u|<\infty$ almost everywhere on $B_{\rho} \times(0, T)$. However, this is an easy consequence of Fatou's lemma and (3.4). Since $\rho>0$ is arbitrary, we can appeal to the classical diagonal argument to conclude the proof.

Lemma 3.4. There exists a subsequence of $\left\{u_{k}\right\}$, still denoted by $\left\{u_{k}\right\}$, and a measurable function $F(x, t)$ on $\Sigma_{T}$ such that

$$
\nabla u_{k} \rightarrow F \quad \text { almost everywhere on } \Sigma_{T} \text {. }
$$


Proof. Fix $\rho>0$, and let $\xi$ be given as in the proof of Lemma 3.3. Assume (3.17) holds. According to Egorov's theorem, for each $\eta>0$ there exists a measurable set $E_{\eta} \subset B_{\rho} \times(0, T)$ such that

$$
\left|B_{\rho} \times(0, T) \backslash E_{\eta}\right|<\eta \quad \text { and } \quad u_{k} \rightarrow u \quad \text { uniformly on } E_{\eta} \text {. }
$$

We may assume that $\left\{u_{k}\right\}$ is bounded in $L^{\infty}\left(E_{\eta}\right)$, and thus by (3.16),

$$
\int_{E_{\eta}}\left|\nabla u_{k}\right|^{p} d x d t \leq c(\eta, \rho)
$$

For $\delta>0$, we can find a $K(\delta)$ with

$$
\left|u_{k}-u_{m}\right|<\delta \text { on } E_{\eta} \text { for all } m, k>K(\delta) \text {. }
$$

Let $P_{\delta}$ be defined as before. We can derive from (3.3a) that

$$
\begin{aligned}
& \frac{d}{d t} \int_{B_{2 \rho}} \int_{0}^{u_{k}(x, t)-u_{m}(x, t)} P_{\delta}(s) d s \xi(x) d x+ \\
& \int_{B_{2 \rho}}\left(\left|\nabla u_{k}\right|^{p-2} \nabla u_{k}-\left|\nabla u_{m}\right|^{p-2} \nabla u_{m}\right)\left(\nabla u_{k}-\nabla u_{m}\right) \xi(x) P_{\delta}^{\prime}\left(u_{k}-u_{m}\right) d x \\
& =-\int_{B_{2 \rho}}\left(\left|\nabla u_{k}\right|^{p-2} \nabla u_{k}-\left|\nabla u_{m}\right|^{p-2} \nabla u_{m}\right) \nabla \xi(x) P_{\delta}\left(u_{k}-u_{m}\right) d x \\
& \leq \frac{\delta}{\rho} \int_{B_{2 \rho}}\left(\left|\nabla u_{k}\right|^{p-1}+\left|\nabla u_{m}\right|^{p-1}\right) d x
\end{aligned}
$$

for $k, m$ sufficiently large. Thus,

$$
\begin{aligned}
& \int_{B_{2 \rho} \times(0, T)}\left(\left|\nabla u_{k}\right|^{p-2} \nabla u_{k}-\left|\nabla u_{m}\right|^{p-2} \nabla u_{m}\right) \\
& \quad \cdot\left(\nabla u_{k}-\nabla u_{m}\right) \xi(x) P_{\delta}^{\prime}\left(u_{k}-u_{m}\right) d x d t \\
& \leq \int_{B_{2 \rho}} \int_{0}^{u_{0 k}-u_{0 m}} P_{\delta}(s) d s d x+\frac{\delta}{\rho} \int_{B_{2 \rho} \times(0, T)}\left(\left|\nabla u_{k}\right|^{p-1}+\left|\nabla u_{m}\right|^{p-1}\right) d x d t \\
& \leq c(\rho) \delta
\end{aligned}
$$

for $k, m$ sufficiently large. We estimate, with the aid of (3.21), (3.22), and (3.23) that

$$
\int_{E_{\eta}}\left|\nabla u_{k}-\nabla u_{m}\right|^{p} d x d t
$$




$$
\begin{aligned}
= & \int_{E_{\eta}} \frac{\left|\nabla u_{k}-\nabla u_{m}\right|^{p}}{\left(\left|\nabla u_{k}\right|+\left|\nabla u_{m}\right|\right)^{\frac{(2-p) p}{2}}}\left(\left|\nabla u_{k}\right|+\left|\nabla u_{m}\right|\right)^{\frac{(2-p) p}{2}} d x d t \\
\leq & \left(\int_{E_{\eta}} \frac{\left|\nabla u_{k}-\nabla u_{m}\right|^{2}}{\left(\left|\nabla u_{m}\right|+\left|\nabla u_{k}\right|\right)^{2-p}} d x d t\right)^{\frac{p}{2}}\left(\int_{E_{\eta}}\left(\left|\nabla u_{m}\right|+\left|\nabla u_{k}\right|\right)^{p} d x d t\right)^{\frac{(2-p)}{2}} \\
\leq & c(\eta, \rho)\left(\int_{B_{2 \rho} \times(0, T)}\left(\left|\nabla u_{k}\right|^{p-2} \nabla u_{k}-\left|\nabla u_{m}\right|^{p-2} \nabla u_{m}\right)\right. \\
& \left.\cdot\left(\nabla u_{k}-\nabla u_{m}\right) \xi(x) P_{\delta}^{\prime}\left(u_{k}-u_{m}\right) d x d t\right)^{\frac{p}{2}} \\
\leq & c_{1}(\eta, \rho) \delta^{\frac{p}{2}}
\end{aligned}
$$

for $k, m$ sufficiently large. We see that $\left\{\nabla u_{k}\right\}$ is a Cauchy sequence in $\left(L^{p}\left(E_{\eta}\right)\right)^{N}$. In particular, we can select a subsequence of $\left\{u_{k}\right\}$, still denoted by $\left\{u_{k}\right\}$, so that

$\nabla u_{k} \quad$ converges almost everywhere on $E_{\eta}$.

This is true for each $\eta>0$, and so $\left\{\nabla u_{k}\right\}$ converges almost everywhere on $B_{\rho} \times(0, T)$. The lemma follows from the classical diagonal argument.

Lemma 3.5. $\left\{\left|\nabla u_{k}\right|^{p-2} \nabla u_{k}\right\}$ is precompact in $L^{1}\left(B_{\rho} \times(0, T)\right)$ for each $\rho>0$.

Proof. Note that the function $G(x) \equiv|x|^{p-2} x$ is continuous because $\lim _{|x| \rightarrow 0}|x|^{p-2} x=0 \equiv G(0)$. Thus, we may assume that

$$
\left\{\left|\nabla u_{k}\right|^{p-2} \nabla u_{k}\right\} \quad \text { converges almost everywhere on } B_{\rho} \times(0, T) .
$$

Now for each $q \in\left(0, \frac{p}{2}\right)$, we can choose $\varepsilon_{0}>0$ so that $q=\frac{1}{2+\varepsilon_{0}} p$. We deduce from (3.4) and (3.15) that

$$
\begin{aligned}
& \int_{B_{\rho} \times(0, T)}\left|\nabla u_{k}\right|^{q} d x d t \\
& =\int_{B_{\rho} \times(0, T)} \frac{1}{\left(1+\left|u_{k}\right|\right)^{\left(1+\varepsilon_{0}\right) \frac{q}{p}}}\left|\nabla u_{k}\right|^{q}\left(1+\left|u_{k}\right|\right)^{\left(1+\varepsilon_{0}\right) \frac{q}{p}} d x d t \\
& \leq\left(\int_{B_{\rho} \times(0, T)} \frac{1}{\left(1+\left|u_{k}\right|\right)^{1+\varepsilon_{0}}}\left|\nabla u_{k}\right|^{p} d x d t\right)^{\frac{q}{p}}
\end{aligned}
$$




$$
\begin{gathered}
\cdot\left(\int_{B_{\rho} \times(0, T)}\left(1+\left|u_{k}\right|\right)^{\left(1+\varepsilon_{0}\right) \frac{q}{(p-q)}} d x d t\right)^{\frac{(p-q)}{p}} \\
\leq c(\rho)\left(\int_{B_{\rho} \times(0, T)}\left(1+\left|u_{k}\right|\right) d x d t\right)^{\frac{(p-q)}{p}} \leq c(\rho) .
\end{gathered}
$$

Since $0<p-1<\frac{p}{2}$, there exists a $q \in\left(p-1, \frac{p}{2}\right)$ such that

$$
\int_{B_{\rho} \times(0, T)}\left|\nabla u_{k}\right|^{q} d x d t \leq c(\rho)
$$

at least for $k$ large enough. This implies that $\left\{\left|\nabla u_{k}\right|^{p-2} \nabla u_{k}\right\}$ is uniformly integrable. This, in conjunction with (3.32) and Vitali's theorem, yields the lemma.

Lemma 3.6. $\left\{u_{k}\right\}$ is precompact in $C\left([0, T] ; L^{1}\left(B_{\rho}\right)\right)$ for each $\rho>0$.

Proof. For $\delta>0$ let

$$
\theta_{\delta}(s)= \begin{cases}1 & \text { if } s>\delta \\ s & \text { if }|s|<\delta \\ -1 & \text { if } s<-\delta\end{cases}
$$

and $\xi$ be given as in the proof of Lemma 3.2. We can conclude from (3.3a) that

$$
\begin{aligned}
& \int_{B_{2 \rho}} \int_{0}^{u_{k}(x, t)-u_{m}(x, t)} \theta_{\delta}(s) d s \xi(x) d x \\
& \quad+\int_{B_{2 \rho} \times(0, t)}\left(\left|\nabla u_{k}\right|^{p-2} \nabla u_{k}-\left|\nabla u_{m}\right|^{p-2} \nabla u_{m}\right) \\
& \cdot\left(\nabla u_{k}-\nabla u_{m}\right) \xi(x) \theta_{\delta}^{\prime}\left(u_{k}-u_{m}\right) d x d \tau \\
& =\int_{B_{2 \rho}} \int_{0}^{u_{0 k}(x)-u_{0 m}(x)} \theta_{\delta}(s) d s \xi(x) d x \\
& -\int_{B_{2 \rho} \times(0, T)}\left(\left|\nabla u_{k}\right|^{p-2} \nabla u_{k}-\left|\nabla u_{m}\right|^{p-2} \nabla u_{m}\right) \theta_{\delta}\left(u_{k}-u_{m}\right) \nabla \xi d x d \tau .
\end{aligned}
$$

Observe that the second integral in (3.27) is nonnegative. Hence, we obtain

$$
\int_{B_{\rho}}\left|u_{k}(x, t)-u_{m}(x, t)\right| d x
$$




$$
\leq \int_{B_{2 \rho}}\left|u_{0 k}-u_{0 m}\right| d x+\left.\frac{1}{\rho} \int_{B_{2 \rho} \times(0, T)}|| \nabla u_{k}\right|^{p-2} \nabla u_{k}-\left|\nabla u_{m}\right|^{p-2} \nabla u_{m} \mid d x d t .
$$

Then the lemma follows from Lemma 3.5.

Lemma 3.7. Let $E \subset \mathbf{R}^{N} \times(0, T)$ be bounded and measurable. Assume that there exists an $M>0$ such that

$$
\left|u_{k}\right| \leq M \quad \text { almost everyshere on } E \text { for } k \text { sufficiently large. }
$$

Then $\left\{\nabla u_{k}\right\}$ is precompact in $\left(L^{p}(E)\right)^{N}$.

Proof. Let $\rho>0$ be such that

$$
B_{\rho} \times(0, T) \supset E
$$

and let $\xi$ be given as in the proof of Lemma 3.2. We conclude from (3.39) that

$$
\begin{aligned}
& \int_{B_{2 \rho}} \xi(x) \int_{0}^{u_{k}(x, t)-u_{m}(x, t)} P_{2 M}(s) d s d x \\
& \quad+\int_{B_{2 \rho} \times(0, T)}\left(\left|\nabla u_{k}\right|^{p-2} \nabla u_{k}-\left|\nabla u_{m}\right|^{p-2} \nabla u_{m}\right) \\
& \quad \cdot\left(\nabla u_{k}-\nabla u_{m}\right) P_{2 M}^{\prime}\left(u_{k}-u_{m}\right) \xi(x) d x d \tau \\
& =\int_{B_{2 \rho}} \xi(x) \int^{u_{0 k}-u_{0 m}} P_{2 M} s d s d x \\
& -\int_{B_{2 \rho} \times(0, T)}\left(\left|\nabla u_{k}\right|^{p-2} \nabla u_{k}-\left|\nabla u_{m}\right|^{p-2} \nabla u_{m}\right) P_{2 M}\left(u_{k}-u_{m}\right) \nabla \xi(x) d x d \tau .
\end{aligned}
$$

Subsequently,

$$
\begin{aligned}
& \int_{E}\left(\left|\nabla u_{k}\right|^{p-2} \nabla u_{k}-\left|\nabla u_{m}\right|^{p-2} \nabla u_{m}\right)\left(\nabla u_{k}-\nabla u_{m}\right) d x d \tau \\
& \leq 2 M \int_{B_{2 \rho}}\left|u_{0 k}-u_{0 m}\right| d x \\
& \quad+\left.\frac{2 M}{\rho} \int_{B_{2 \rho} \times(0, T)}|| \nabla u_{k}\right|^{p-2} \nabla u_{k}-\left|\nabla u_{m}\right|^{p-2} \nabla u_{m} \mid d x d t .
\end{aligned}
$$

A calculation similar to (3.24) yields

$$
\begin{aligned}
& \int_{E}\left|\nabla u_{k}-\nabla u_{m}\right|^{p} d x d t \\
& \leq c(M, \rho)\left(\int_{B_{2 \rho}}\left|u_{o k}-u_{0 m}\right| d x\right.
\end{aligned}
$$




$$
\left.+\left.\int_{B_{2 \rho}}|| \nabla u_{k}\right|^{p-2} \nabla u_{k}-\left|\nabla u_{m}\right|^{p-2} \nabla u_{m} \mid d x d t\right)^{\frac{p}{2}} .
$$

This implies the desired result.

Now we are ready to conclude the proof of Theorem 3.1. Let $\left\{v_{k}\right\},\left\{u_{k}\right\}$ be given as before. Note from Lemma 2.3 that

$$
\begin{array}{rlll}
v_{k} \leq v_{k+1} & \text { on } & \Sigma_{T} & \text { for all } k \\
w_{k} \geq w_{k+1} & \text { on } & \Sigma_{T} & \text { for all } k .
\end{array}
$$

Define

$$
\begin{aligned}
v(x, t) & =\lim _{k \rightarrow \infty} v_{k}(x, t), \\
w(x, t) & =\lim _{k \rightarrow \infty} w_{k}(x, t) .
\end{aligned}
$$

Consequently,

$$
w \leq u_{k} \leq v \quad \text { almost everywhere. }
$$

By a result in $[\mathbf{D H}]$, there holds

$$
\int_{s}^{T} \int_{B_{\rho}} \frac{\left(z_{t}\right)^{2}}{(|z|+1)^{1+\varepsilon}} d x d t \leq c(\varepsilon, s, p), T>s>0, \varepsilon>0, \rho>0,
$$

where $z=w$ or $v$. The remaining proof is entirely similar to that in [X1]. The only difference is that in (3.23) of [X1] we require

$$
\varphi \in C_{0}^{\infty}\left(\mathbf{R}^{N} \times(-\infty, T)\right)
$$

This completes the proof.

Acknowledgment: This work was supported in part by the Arkansas Science \& Technology Authority 94-B-18. The author would like to thank Prof. E. DiBenedetto for his interest in this work.

\section{References}

[BM] L. Boccardo and F. Murat, Almost everywhere convergence of the gradients of solutions to elliptic and parabolic equations, Nonlinear Anal., 19 (1992), 581-597.

[BGDM] L. Boccardo, T. Gallouet, J.I. Diaz and F. Murat, Existence and regularity of renormalized solutions for some elliptic problems involving derivatives of nonlinear terms, J. Differential equations, to appear.

[D] E. DiBenedetto, Degenerate Parabolic Equations, Springer-Verlag, New York, 1993. 
[DH] E. DiBenedetto and M.A. Herrero, Non-negative solutions of the evolution p-Laplacian equation, initial traces, and Cauchy problem when $1<p<2$, Arch. Rat. Mech. Anal., 111 (1990), 225-290.

[DL1] R.J. DiPerna and P.L. Lions, Globale existence for the Fokker-Planck-Boltzmann equations, Comm. Pure Appl. Math., 11 (1989), 729-758.

[DL2] - On the Cauchy problem for Boltzmann equations: globale existence and weak stability, Ann. Math., 130 (1989), 321-366.

[LSU] O.A. Ladyzenskaya, V.A. Solonnikov and N.N. Uralceva, Linear and Quasilinear equations of Parabolic Type, AMS, Rhode Island, 1968.

[O] J.T. Oden, Qualitative Methods in Nonlinear Mechanics, Prentice-Hall, Inc., New Jersey, 1986.

[S] J. Simon, Compact sets in the space $L^{p}(0, T, B)$, Am. Mat. Pura Appl., 146 (1987), 65-96.

[X1] X. Xu, On the initial-boundary-value problem for $u_{t}-\operatorname{div}\left(|\nabla u|^{p-2} \nabla u\right)=0$, Arch. Rational Mech. Anal., 127 (1994), 337-360.

[X2] T. Kilpeläinen and X. Xu, On the uniqueness problem for quasilinear elliptic equations involving measures, Revista Mat. Iberoamericana, 12 (1996), to appear.

Received November 23, 1993.

MississipPi State UNIVERSITY

MississipPi STAE, MS 39762

E-mail address: xxu@math.msstate.edu 



\section{PACIFIC JOURNAL OF MATHEMATICS}

Volume $174 \quad$ No. $1 \quad$ May 1996

A distance formula for algebras on the disk

CHRISTOPHER J. BISHOP

Rigidity of isotropic maps

FERNANDO CUKIERMAN

The Schwartz space of a general semisimple Lie group. V. Schwartz

class wave packets

REBECCA A. HERB

Rational polynomials with a $\mathbf{C}^{*}$-fiber

SHULIM KALIMAN

Linear combinations of logarithmic derivatives of entire functions with 195 applications to differential equations

Joseph B. Miles and JoHn Rossi

Factorization problems in the invertible group of a homogeneous $C^{*}$-algebra

N. Christopher PHiLlips

Higher order estimates in complex interpolation theory

RICHARD ROCHBERG

Braid commutators and Vassiliev invariants

TED STANFORD

On the Cauchy problem for a singular parabolic equation 